\title{
Comparison between innovative techniques of photogrammetry
}

\author{
Vincenzo Barrile ${ }^{1, *}$, Giuliana Bilotta $^{2}$, and Alice Pozzoli ${ }^{3}$ \\ ${ }^{1}$ DICEAM Department, Faculty of Engineering Mediterranean University of Reggio Calabria, 89100 Reggio Calabria, Italy \\ ${ }^{2}$ Department of Planning, IUAV University of Venice, 30135 Venice, Italy \\ ${ }^{3}$ DICA Department, Politecnico di Milano, 20133 Milan, Italy
}

\begin{abstract}
Main objective of this work is to suggest some easy procedures to solve typical non-linear problems of analytical photogrammetry, which are also frequent in digital photogrammetry. In particular, we propose a two-steps procedure based on two phases: Relative Orientation and Absolute Orientation, both characterized by non-linear functions. It is firstly necessary to form the model, finding the solution of the Relative Orientation, and then to reconstruct the object considering the solution of the Absolute Orientation. Once validated the technique of orientation of two images, a procedure for the orientation of three images is proposed. In particular, the introduction of a third image allows avoiding human decision to find the final solution. In order to find an easy solution for three-image orientation, each model coming from two images of a triplet is analysed, computing the relative orientation bby using an exhaustive research of preliminary values of its parameters. This non-conventional approach supplies the orientation of two images, taking into account a priori information among four base solutions. Once each model is relatively registered, the absolute orientation is computed by using a linear parameterization. We did a comparison between the method of the three photogrammetric images and a method of Computer Vision.
\end{abstract}

\section{Introduction}

The main function of photogrammetry is the transformation of data from the image space to the object space. This transformation can be made in a direct way, with collinearity equations, or in two steps, with the formation of a model and, only in a second time, with the reconstruction of the original object [1].

It is well known that model formation and object reconstruction require the solution of the problems of Relative Orientation and Absolute Orientation respectively, which have both non-linear functional models. Therefore, they need respectively an exhaustive research of the preliminary values of parameters for the model formation, and a transformation of the parameters able to transform the problem of the object reconstruction in a simple linear problem. This way of working makes the orientation procedures much more flexible, and allows wide applications in close range photogrammetry, which is actually growing in importance.

\section{From images to object via model}

In the procedure of object reconstruction, it is necessary to take into account that at least two images are needed for reconstructing an object. In particular, a relation of roto-translation with scale variation constitutes the link between the coordinates of the point $\mathrm{Q}(\mathrm{x}, \mathrm{y}, \mathrm{z})$ in an image, and the coordinates of the corresponding point $\mathrm{P}$ $(\mathrm{X}, \mathrm{Y}, \mathrm{Z})$ in the object space. Both reference systems are traditionally Cartesian reference systems, but it is possible to achieve the same results, with minor changes, using a different reference system, suitably linked to the previous ones. Let us show the above-mentioned relation:

$$
\mid \begin{gathered}
x^{\circ} \\
y^{\circ} \\
-\left.c\right|_{i j}
\end{gathered}=\hat{\lambda}_{i j} \hat{R}_{j}\left(\left|\begin{array}{c}
\hat{X} \\
\hat{Y} \\
\hat{Z}
\end{array}\right|_{i}-\left|\begin{array}{c}
\hat{X}_{0} \\
\hat{Y}_{0} \\
\hat{Z}_{0}
\end{array}\right|\right)
$$

Where, as shown in Fig. 1:

$\mathrm{x}^{0}, \mathrm{y}^{0}, \mathrm{c}=$ image coordinates and principal distance

$\hat{\mathrm{X}}_{0}, \hat{\mathrm{Y}}_{0}, \hat{Z}_{0}=$ coordinates of projection center

$\hat{\mathbf{X}}, \hat{\mathbf{Y}}, \hat{\mathbf{Z}}=$ object coordinates

$\lambda=$ scale factor, variable point by point

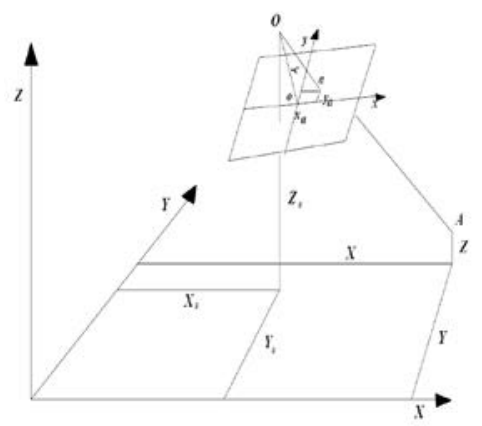

Fig. 1. Reference photogrammetric systems.

\footnotetext{
Corresponding author: vincenzo.barrile@unirc.it
} 
The photogrammetric technique is based on a transformation of a perspective (or a couple of perspectives) in a quoted orthogonal projection. In this transformation, we have non-linear equations and, before starting the plotting, we need information about the preliminary value of unknown parameters. Our main aim is to find expressions working with parameters easy to be obtained. In order to orient two images in the $3 \mathrm{D}$ space, we can choose two different procedures: a "one step" or a "two steps". The first procedure is based on collinearity equations and needs 12 parameters: $\mathrm{X}_{1}, \mathrm{Y}_{1}$, $Z_{1}, X_{2}, Y_{2}, Z_{2}$ (coordinates of the two projection centers), and $\omega_{1}, \varphi_{1}, \kappa_{1}, \omega_{2}, \varphi_{2}, \kappa_{2}$ (attituded angles of the two sensor). The second one separates the model formation (Relative Orientation) from the object reconstruction (Absolute Orientation). In this procedure, we define the problem of Relative Orientation by means of 5 parameters: $\varphi_{1}, \kappa_{1}, \omega_{2}, \varphi_{2}, \kappa_{2}$ (Symmetric Relative Orientation), or $b_{y}, b_{z}, \omega_{2}, \varphi_{2}, \kappa_{2}$ (Asymmetric Relative Orientation). On the contrary, to define the problem of Absolute Orientation we need 7 parameters: $t_{x}, t_{y}, t_{z}$ (shift vector), $\lambda$ (scale factor), $\Omega, \Phi, \mathrm{K}$ (Cardanic angles).

\section{Model construction}

\subsection{Relative Orientation Parameters}

Regarding the Relative Orientation, we make an exhaustive research of the preliminary values, solving a linearized problem in all its possible cases. Notice that an exact solution has been found, but it leads to an equation of order four, which supplies four plausible solutions, as we can easily achieve by repeating a linearized problem via an exhaustive research.

The Relative Orientation is based on the Coplanarity Condition. It shows that the point $\mathrm{P}_{1}$, in the first image, and its homologue point $\mathrm{P}_{2}$, in the second image, have a unique corresponding point $\mathrm{Q}$ on the object.

In case of Asymmetric Relative Orientation, we have to define $b_{y}, b_{z}, \omega_{2}, \varphi_{2}, \kappa_{2}$, which are the parameters of position and attitude of the second image, compared to those of the first image. Notice that $b_{x}$ is already defined in the Absolute Orientation, as the scale factor $\lambda$. In case of Symmetric Relative Orientation, we have to define $\varphi_{1}$, $\kappa_{1}, \omega_{2}, \varphi_{2}, \kappa_{2}$, parameters which represent position and attitude of the two in the Absolute Orientation, as the global attitude angle $\Omega$.

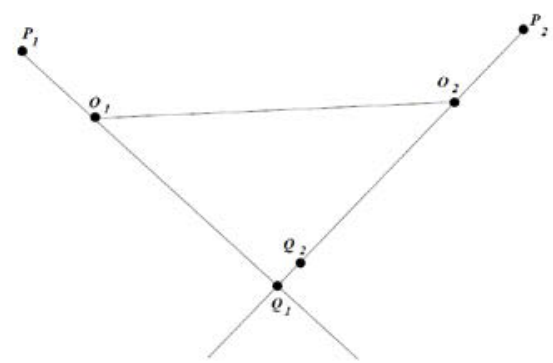

Fig. 2. Coplanarity condition.

\subsection{Exhaustive Research}

For the Relative Orientation, we should have previous information about the preliminary values of the parameters. It is not always possible to know them, before the plotting. Let us point out that nonconventional photogrammetry implies often camera acquisition without classical surveying measurement. If we consider the classical Symmetric procedure of Relative Orientation, we can make an exhaustive research of all possible preliminary parameters, because we work in a closed group (in the topological sense) of values compared to the rotations in the space.

Notice that in the Asymmetric procedure of Relative Orientation, we have two shift parameters to be searched, but the group of shifting is not a closed one, so we had to use a different way to find the preliminary values. However with the following relations is possible to transform the Symmetric Relative Orientation parameters in the Asymmetric ones, and vice versa:

$\begin{array}{ll}b_{x}=\cos \varphi_{1} \cos k_{1} & \varphi_{1}=\arcsin b_{z} \\ b_{y}=\cos \varphi_{1} \sin k_{1} & k_{1}=\operatorname{arcatn} \frac{b_{y}}{b_{x}} \\ b_{z}=\sin \varphi_{1} & \end{array}$

$$
\begin{aligned}
& R_{2}^{T}\left(\varpi_{2} \varphi_{2} k_{2} \mid b_{x} b_{z}\right)=R_{2}^{T}\left(\varpi_{2} \varphi_{2} k_{2}\right) R_{1}\left(\varpi_{1} \varphi_{1} k_{1}\right) \\
& R_{2}^{T}\left(\varpi_{2} \varphi_{2} k_{2}\right)=R_{2}^{T}\left(\varpi_{2} \varphi_{2} k_{2} \mid b_{x} b_{z}\right) R_{1}^{T}\left(\varpi_{1} \varphi_{1} k_{1}\right)
\end{aligned}
$$

The convergence of linearization of trigonometric functions is acceptable as far as values lower or near $\Pi / 4$. Therefore, we decided to explore all the admissible values for rotation angles with a step of $\Pi / 4$, as shown in Table 1.

As known, if the $\varphi$ angle is around $\pm \Pi / 2$, we cannot individuate the $k$ rotation, which is fixed equal to zero. Indeed in the polar zones (we assumed their range in a circle of one degree), the two angles are identical or quasi identical, and this fact produced singularity or illconditioning.

Table 1. Exhaustive Research for Symmetric Relative Orientation parameters.

\begin{tabular}{|c|c|c|c|c|c|}
\hline & $\varphi_{1}$ & $k_{1}$ & $\omega_{2}$ & $\varphi_{2}$ & $k_{2}$ \\
\hline$\Pi / 2$ & $\circ$ & & & $\circ$ & \\
\hline$\Pi / 4$ & $\bullet$ & & & $\bullet$ & \\
\hline 0 & $\bullet$ & $\bullet$ & $\bullet$ & $\bullet$ & $\bullet$ \\
\hline$\Pi / 4$ & $\bullet$ & $\bullet$ & $\bullet$ & $\bullet$ & $\bullet$ \\
\hline$\Pi / 2$ & $\circ$ & $\bullet$ & $\bullet$ & $\circ$ & $\bullet$ \\
\hline $3 \Pi / 4$ & & $\bullet$ & $\bullet$ & & $\bullet$ \\
\hline$\Pi$ & & $\bullet$ & $\bullet$ & & $\bullet$ \\
\hline $5 \Pi / 4$ & & $\bullet$ & $\bullet$ & & $\bullet$ \\
\hline $3 \Pi / 2$ & & $\bullet$ & $\bullet$ & & $\bullet$ \\
\hline $7 \Pi / 4$ & & $\bullet$ & $\bullet$ & & $\bullet$ \\
\hline
\end{tabular}

Where ${ }^{\circ} \mathrm{k}_{1} \equiv 0$ if $\varphi_{1} \equiv \pm \Pi / 2$ and/or $\mathrm{k}_{2} \equiv 0$ if $\varphi_{2} \equiv \pm \Pi / 2$ 
The exhaustive research explored $5 \times 8 \times 8 \times 5 \times 8=128$ possible configurations. For each case, a linear system was solved, using the values of this configuration (case), as preliminary values of the parameters of the Symmetric Relative Orientation.

Examples were carried out in all the middle points of the possible configuration. Considering the 5 parameters of the Symmetric Relative Orientation, the angles $\kappa_{1}, \omega_{2}$, $\kappa_{2}$ are defined in a complete rotation (8 configurations), whilst $\varphi_{1}, \varphi_{2}$ are defined in a half rotation (5 configurations), which led to the above mentioned 12800 cases.

Each linear system solution gave us the estimate parameters for the Symmetric Relative Orientation. The convergence to admissible values is when $\sigma_{0}$ is small enough. Considering only the distinct solutions, we found four analytical acceptable configurations.

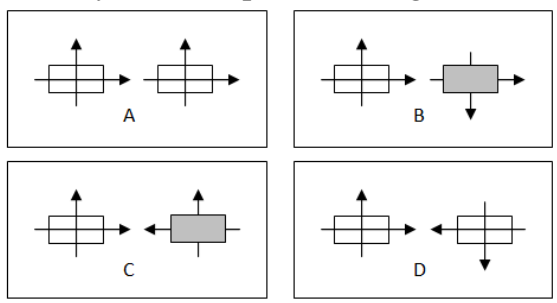

Fig. 3. The four final possible configurations.

\section{Object reconstruction}

\subsection{Absolute Orientation}

Starting from a rototraslation in the space, a rational alternative to classical Rotation Matrix is the Rodriguez Matrix.

$$
\mathbf{R}=(\mathbf{I}-\mathbf{S})^{-1}(\mathbf{I}+\mathbf{S})
$$

Where $\mathbf{I}$ is the identity matrix of $3 \times 3$ dimensions, and $\mathbf{S}$ is an emisymmetric matrix defined as follows:

$$
\mathbf{S}=\left|\begin{array}{ccc}
0 & c & -b \\
-c & 0 & a \\
b & -a & 0
\end{array}\right|
$$

This emisymmetric matrix $\mathbf{S}$ permits to find the exact solution of the absolute orientation problem, thanks to the solution of a linear system, after a suitable substitution of variables. We start from the conventional rototraslation in the space, with a global scale variation $\lambda$, and we compute the expected value in a way to eliminate the shift vector $t$ :

$$
\begin{aligned}
& y_{i}=\lambda R_{i} x+t \\
& \bar{y}=\lambda R \bar{x}+t \\
& y_{i}-\bar{y}=\lambda R\left(x_{i}-\bar{x}\right)
\end{aligned}
$$

Indeed, it is possible to calculate $t$ lately with the following expression:

$$
t=\bar{y}-\lambda R \bar{x}
$$

Making the square of the second expression in the formula number (11), we also find an easy expression to calculate the scale factor:

$$
y^{T} y=\lambda^{2} x^{T} R^{T} R=\lambda x^{2} x^{T} x \Rightarrow \lambda=\sqrt{\frac{y^{T} y}{x^{T} x}}
$$

Now we have to make a substitution of variables in a way to transform the non-linear problem in a linear one:

$$
y_{i}=\lambda R x_{i}
$$

With a further substitution, we obtain our linear system of equations:

$$
y_{i}=R \sqrt{\frac{y^{T} y}{x^{T} x}} x_{i}=R{ }_{i} x
$$

Thus, after these simple substitutions, we obtain a linear solution, showing the direct proportion between the model coordinates $x=x\left(u^{\circ}, v^{\circ}, w^{\circ}\right)$ and the object ones $y=y(X, Y, Z)$ :

$$
\begin{aligned}
& y_{i}=R_{i}=(I x-S)^{-1}(I+S) x_{i} \Rightarrow(I-S) y_{i}=(I+S) x_{i} \\
& \left.\left|\begin{array}{ccc||c|c|c}
1 & -c_{j} & b_{j} \\
c_{j} & 1 & -a_{j} \\
-b_{j} & a_{j} & 1
\end{array}\right| \begin{array}{|ccc}
\hat{X} \\
\hat{Z}
\end{array}\right|_{i}=\left.\left|\begin{array}{ccc}
1 & c_{j} & -b_{j} \\
-c_{j} & 1 & a_{j} \\
b_{j} & -a_{j} & 1
\end{array}\right| \begin{array}{c}
u^{\circ} \\
v^{\circ} \\
w^{\circ}
\end{array}\right|_{i}
\end{aligned}
$$

Reorganizing matrices and vectors, in a way which collects in a unique vector the three unknown parameters, coming from the above mentioned emisymmetric matrix, we obtain the following final equation:

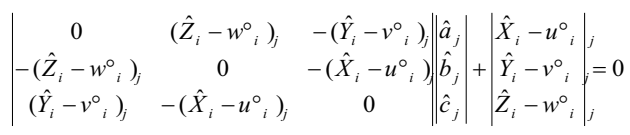

\subsection{Exact Solution of the Absolute Orientation}

In our procedure for the Absolute Orientation, the object reconstruction does not need preliminary parameters, because we can reach the exact solution, by solving the linear system, mentioned in the above paragraph. We tested this procedure, considering 208 possible configurations. These cases come from an object rotation following the global attitude angles $(\Omega, \Phi, \mathrm{K})$, with a step of $\Pi / 4$. Exam was performed analyzing the rotation in the space of a cube with 27 control points, regularly distributed.

\section{Three-images procedure}

\subsection{Three-image orientation through exhaustive research}

In the following, an automatic procedure of external orientation of three images will be proposed. This procedure doesn't require previous knowledge of the unknown orientation parameters. Indeed, exploring the $3 \mathrm{D}$ space with a step of $\Pi / 4$, it is possible to find all the preliminary values of the unknown orientation parameters. This idea want to avoid the linearization of the orientation functions supplying the lack of 
information about the position and the attitude of an image. The procedure is based on the classical two-step approach of photogrammetry, i.e. relative and absolute orientation. References [2] and [3] show a solution of relative orientation problem based on exhaustive research of the preliminary values of parameters proposed. First, given a tern of images, all possible combinations of pairs of images are considered; in such a way, their relative orientation is computed, by using an exhaustive research of the approximate values of the unknown parameters. By using this unconventional approach, each pair of images gives four analytically acceptable solutions, among which, only the decision of the user would allow to select the proper one. Actually, to start the Absolute Orientation, we have to select manually one among the founded four configurations. The idea of introducing a third image allows to avoid human intervention to find the final solution. In fact, after obtaining the four possible solutions related to each pair of images, it is necessary to perform a linkage between the models. By following this procedure, sixteen possible combinations are obtained; however, only two of these combinations, the specular ones, are consistent with the observed object. Finally, the searched solution is obtained by orienting the two possible solutions in an absolute reference system.

\subsection{Bridging the models}

The step of the Model Construction gives four admissible solutions, as above said, and produces four distinct models (called A, B, C, D). In case of three partially overlapped images, this step can be repeated two times. Indeed the model 1-2 can be formed by the images 1 and 2, and the model 1-3 can be formed by the images 1 and 3. A 3D S-transformation allows to make the bridging of these models, taking into account all the four models obtained according to the admissible solutions founded in the Relative Orientation. The procedure leads to sixteen different small blocks.

The majority of these blocks are completely unlikely; indeed the sigma naught of the 3D S-transformation adjustment is enormous. This fact is reasonable because if and only if both models (1-2 and 1-3) are congruent between themselves, the bridging can be carried out successfully.

\section{Numeric experiments}

To verify precision, accuracy and reliability of these techniques, a program in Fortran 95 language (compiled and assembled with Lahey-Fujitsu Fortran 95 version 5.6) was written, implemented and tested. In all the examples, we introduced random errors, with standard deviation of $20 \mu \mathrm{m}$, as usual in photogrammetry. In the following flowchart (Fig. 4), we will summarize the global procedure for the orientation of two images, then we will present a brief explanation of these programs.

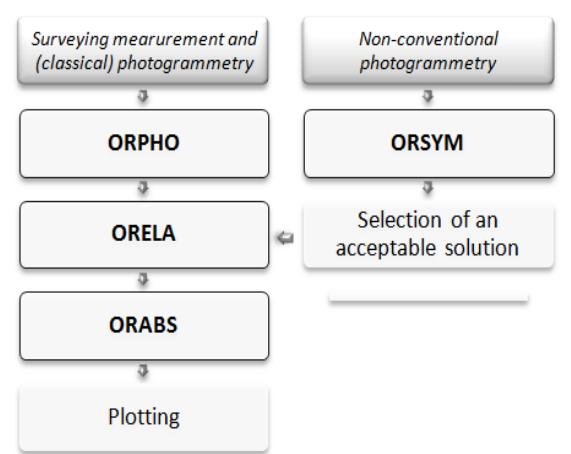

Fig. 4. Global procedure for the orientation of two images.

$\mathrm{ORPHO}_{-}$it converts Cardanic angles in Eulerian angles and viceversa. This is a very large transformation used in close range photogrammetry, because it is essential for the image orientation, when the rotation angles are acquired by surveying measurements.

ORSYM_ it calculates the preliminary values for the Symmetric Relative Orientation. It solves 12800 linear problems, exploring all possible configurations in the space, with a step of $\Pi / 4$. The same program, choosing one of the four distinct solutions, allows to calculate the preliminary parameters for the Asymmetric Relative Orientation.

ORELA_ it calculates the adjusted parameters of the Asymmetric Relative Orientation, starting from its preliminary ones. If these preliminary values are unknown, at the data acquisition, it is possible to get them from the results of the previous program. On the contrary, if they are already known, it is possible to transform the Eulerian angles into the Cardanic ones, by means of ORPHO.

ORABS _ it calculates the adjusted Absolute Orientation parameters. They are calculated with a simple substitution of variables, able to transform the non-linear problem of the Absolute Orientation in a linear one.

Before to conclude we wish to presents some results of these experiments. We considered robust statistical index (mode, median, $1^{\text {st }}$ and $3^{\text {rd }}$ quantiles), able to analyze distribution free problems.

The following tables and figures show the difference among the nominal values and the preliminary ones.

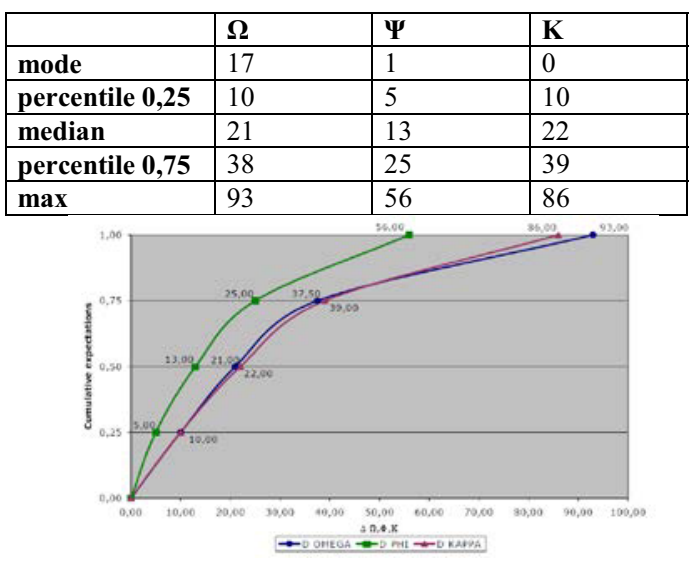

Fig. 5. Absolute Orientation Results. 


\begin{tabular}{|l|l|l|l|l|l|}
\hline & $\boldsymbol{\Delta} \boldsymbol{\varphi}_{\mathbf{1}}$ & $\boldsymbol{\Delta} \boldsymbol{\kappa}_{\mathbf{1}}$ & $\mathbf{\Delta} \boldsymbol{\omega}_{\mathbf{2}}$ & $\mathbf{\Delta} \boldsymbol{\varphi}_{\mathbf{2}}$ & $\mathbf{\Delta} \boldsymbol{\kappa}_{\mathbf{2}}$ \\
\hline mode & 8 & 5 & 8 & 7 & 7 \\
\hline percentile 0,25 & 18 & 11 & 33 & 16 & 22 \\
\hline median & 46 & 41 & 91 & 36 & 53 \\
\hline percentile 0,75 & 84 & 99 & 176 & 68 & 139 \\
\hline max & 287 & 569 & 937 & 286 & 827 \\
\hline
\end{tabular}

Fig. 6. Symmetric Relative Orientation results.

\begin{tabular}{|l|l|l|l|l|l|}
\hline & $\mathbf{\Delta} \boldsymbol{\varphi}_{\mathbf{1}}$ & $\mathbf{\Delta} \boldsymbol{\kappa}_{\mathbf{1}}$ & $\mathbf{\Delta} \boldsymbol{\omega}_{\mathbf{2}}$ & $\mathbf{\Delta} \boldsymbol{\varphi}_{\mathbf{2}}$ & $\mathbf{\Delta} \boldsymbol{\kappa}_{\mathbf{2}}$ \\
\hline mode & 96 & 491 & 208 & 14 & 275 \\
\hline percentile 0,25 & 36 & 224 & 362 & 19 & 250 \\
\hline median & 68 & 432 & 760 & 47 & 514 \\
\hline percentile 0,75 & 102 & 682 & 1301 & 98 & 862 \\
\hline max & 165 & 2205 & 2937 & 251 & 1895 \\
\hline
\end{tabular}

Fig. 7. Symmetric Relative Orientation results (Polar regions).

In the light of the proposed experiments, new programs, which would permit an easy solution for the Relative and Absolute Orientations, gave very satisfied expected results. This procedure has great potentiality for non-conventional data acquisition (e.g. amatorial images, images coming from unknown and old sources, equipped vehicles, robots, and many other applications in close range photogrammetry). The advantage of a linear Absolute Orientation should also taken into account. Moreover, even if the solution achieved in the Relative Orientation requires an exhaustive research, it is again quick and easy, and seems to solve positively the problem how to acquire the preliminary values of the Relative Orientation parameters.

\section{A global procedure for 3 images orientation}

As above shown, the global procedure for the orientation of two images can be summarized in the flowchart of Fig. 4. With the new procedure, we eliminate any human intervention after the starting inputs. For that reason we unify all the Orientation programs in one called ORTRE. This program can run automatically and is able to find the adjusted parameters of the Absolute Orientation.

In the following flowchart (Fig. 8) we want to show how all the global procedure run after the starting inputs of three images which is the same as for the orientation of two images.

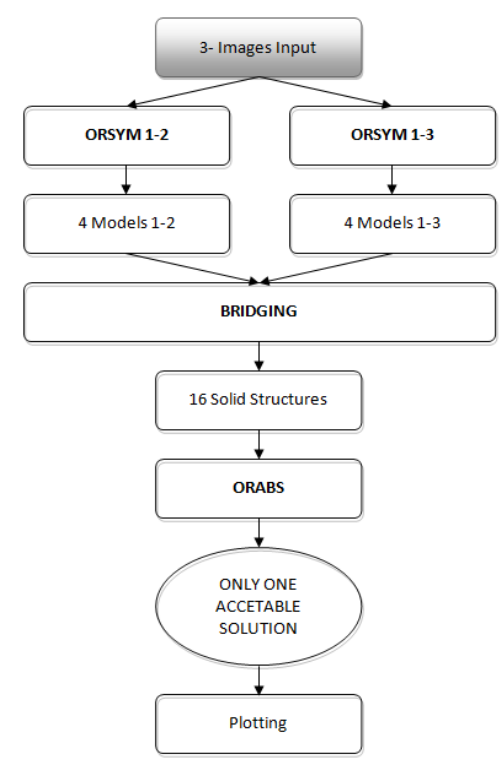

Fig. 8. Image Orientation - Global Proced. (ORTRE Program).

\section{Computer Vision}

\subsection{Method of Computer Vision}

Regarding the second method, we present the results of testing and comparison between the two methods applied and implemented.

Objective of the work is to estimate, more precisely as possible, a $3 \mathrm{~d}$ model of marine surfaces. Specifically, we presented the same innovative proposal - rigorous photogrammetry type that makes use of three frames acquired simultaneously from different locations - and a methodology of Computer Vision.

The advantages of the Computer Vision technique, as is known, are low costs, automaticity and immediacy: we can get an accurate and usable result for further processing and analysis processes. Following the acquisition of the images, the reconstruction of the 3D model from digital images was performed with commercial software, based on special algorithms of the Structure from Motion technique (SfM). The SfM is successfully used in a wide range of applications, for generation of photogrammetry, survey and topographical mappings, to control activities, for the evaluation of damage due to disasters as well as for the preservation of evidence for legal disputes resolutions.

\subsection{Application and study area}

While waiting to comparing a hydraulic model confluence already experienced in Milan, we present the results of experiments made comparing, on a sea area of Reggio Calabria [4], the model obtained through the methodology of Computer Vision with the model obtained from the rigorous photogrammetric technique with three cameras.

The software used was PhotoScan in the Professional version 1.2.4, developed by Agisoft House LLC of St. 
Petersburg (Russia). For imaging we used a HP Z800 workstation, with two CPU Xeon Hexa Core X5650, 64 GB Ram, mass storage 128 GB SSD and additional Hard Disk 2TB, two video cards NVIDIA Quadro FX 4800, each with 1, 5 GB RAM, and OS Windows 7 Professional 64-bit. The computerized processing procedure to a 3D model frames took place in a specific order in different phases. The first phase was the alignment of the images and the construction of the sparse point cloud (cloud points basis). This step identifies the common points in both frames using the image matching algorithms. The points selected in the various photos must necessarily have some features in common to be able to be properly overlapped. The result is an overall view of all shooting points positioned along the three Cartesian axes (local reference system) and the points constituting the cloud of anchorage points. The construction of the denser point cloud (dense cloud points) represents the second stage of processing, in which starting from the cloud of sparse points we get a more detailed and dense cloud of points.

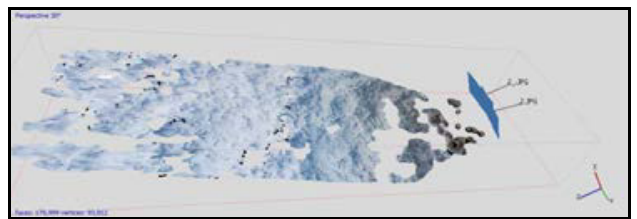

Fig. 9. Texture obtained with point shooting.

The 3D model with texture can be scaled and georeferenced using the data collected during the campaign of shooting and establishing, on the frames and on the model of those markers points, for scaling the model. For validating results obtained in experiments carried out by the use of the pair of digital cameras and commercial software Agisoft PhotoScan (a "low-cost model"), we realized a metric three-dimensional model of the sea surface, in the same area and at the same moment, experiencing the use of three digital cheap camcorders resistant to weathering NILOX Mini Action Cam. We placed devices triangularly at equal distances of $1 \mathrm{~m}$, performing the processing of the acquired data using the rigorous and experimental photogrammetric methodology with three cameras already exposed in the preceding paragraphs of the article.

In terms of accuracy, estimation of the leading photogrammetric orientation parameters, accuracy of results, we found a substantial similarity of results of the two models.

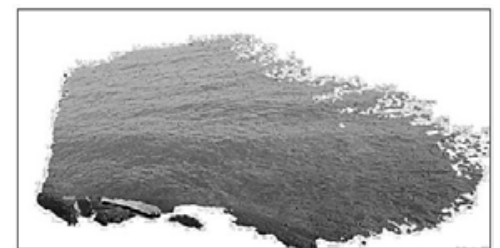

Fig. 10. 3D model (rigorous photogrammetric technique).

Table 2. Comparison of the values obtained from the two models

\begin{tabular}{ccc}
\hline & $\Delta[\mathrm{m}]$ & $\sigma[\mathrm{m}]$ \\
Technique 1 & 0.05 & 0.07 \\
Technique 2 & 0.03 & 0.06 \\
\hline
\end{tabular}

Fig. 11 shows comparisons between the two techniques in terms of accuracy obtained by setting the target positioned on the homologous elements, such as the edges of the reef and the "test" fixed points arranged appropriately on the sea surface.

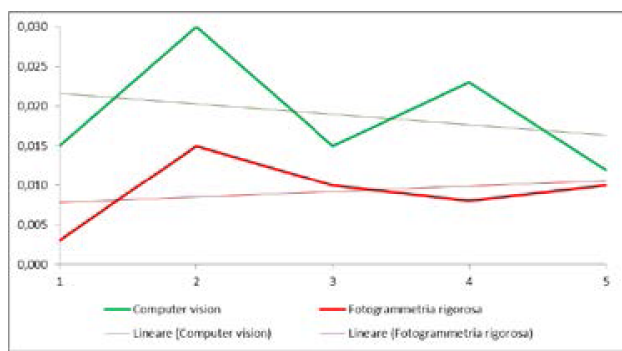

Fig. 11. Comparison of the results obtained by the two techniques.

\section{Conclusions, further developments}

We proposed a solution to solve for the problem of object reconstruction from three images not requiring any initial approximations for orientation parameters. This first procedure is based on the classical two steps approach of photogrammetry, i.e. relative and absolute orientation. All possible combinations of pairs of images in the triplet are considered and relatively registered by an algorithm running on exhaustive research [2], [3].

Advantages of this method are twofold. First, the three-image approach is based on a more reliable configuration with respect to simple relative orientation of a pair of images. Secondly, the method is very suitable to provide initial values of the unknowns to solve for machine vision or complex photogrammetric problems. This way of working makes the orientation procedures much more flexible, and allows wide applications in close range photogrammetry, which is actually growing in importance. Indeed not only classical close range photogrammetry, (e.g. for architectural and archaeological surveying as well as for industrial applications), but also data acquisition by equipped vehicles, and robots are nowadays typical data, largely diffused. Especially in the last cases, the need of real time (or quasi-real time) data processing and validation is very high. For these reasons, model formation and object reconstruction require the solutions of the problems of Relative Orientation and Absolute Orientation respectively, avoiding to waste time in an "a posteriori" search of preliminary values. Having regards to the promising results obtained, we may experience the technique of Computer Vision also on the same study area described in the previous sections of this paper.

\section{References}

1. K. Kraus, Photogrammetry. Bonn: Dümmler, 1, 2 (1993)

2. L. Mussio, A. Pozzoli, "Non-Linear Problems of Analytical Photogrammetry", IAPRS, 34, Part 6/W11, pp. 210-215 (2003)

3. L. Mussio, A. Pozzoli, "Quick Solutions 
particularly in Close Range Photogrammetry", IAPRS, 34, Part 6/W12, pp. 273-278 (2003)

4. V. Barrile, D. Lamari, V. Gelsomino, "Tecniche imaging per la modellazione $3 \mathrm{D}$ della superficie marina", Atti del $61^{\circ}$ Convegno nazionale SIFET, Lecce (2016)

5. Y.L. Abel-Aziz, H.M. Karara, "Direct Linear Transformation into Object Space Coordinates in Close-Range Photogrammetry", Proc. of Symp. on Close-Range Photogramm., pp. 1-18 (1971)

6. S. Finsterwalder, "Die Geometrischen Grundlagen der Photogrammetrie", Jahresbericht der Deutschen Mathematiker-Vereinigung, 6, Teubner, Leipzig (1899)

7. H.G. Fourcade, "A new method of aerial surveying”, Trans. Roy. Soc. of South Africa, 14, 1, pp. 93-112 (1926)

8. S. Hattori, Y. Myint, "Automatic Estimation of Initial Approximations of Parameters for Bundle Adjustment", $P E \& R S, 61,7$, pp. 909-915 (1995)

9. C. Heipke, "Automation of Interior, Relative and Absolute Orientation", ISPRS J Photogramm Remote Sens., 52, pp. 1-19 (1997)

10. H.-P. Pan, "A Direct Closed-Form Solution to General Relative Orientation", Technical Report on Photogrammetron., pp. 1-20 (1996)

11. E. Kruppa., "Zur Ermittlung eines Objektes aus zwei Perspektiven mit inner Orientierung", Sitz.Ber. Akad. Wiss., Wien, Math. Naturw. Kl. (Abt. IIa 122), pp. 1939-1948 (1913)

12. H. C. Longuet-Higgins, "A computer algorithm for reconstructing a scene from two projections", Nature, 293, 10, pp. 133-135 (1981)

13. H.-P. Pan, "A direct closed-form solution to general relative orientation of two stereo views", Digit. Sign. Process., 9, 3, Academic Press, pp. 195-211 (1999)

14. T. Sarjakoski, "Concept of a completely digital stereoplotter", The Photogramm. Journ. of Finland, 2, pp. 95-100 (1981)

15. H. Schmid, "An analytical treatment of the orientation of a photogrammetric camera", Photogr. Eng., 20, pp. 765-781 (1954)

16. G. Schut, "Analytical aerial triangulation and comparison between it and instrumental aerial triangulation", Photogrammetria, 12, pp. 311-318 (1955-56)

17. P. Stefanovic, "Relative Orientation - a new approach", ITC Journal, pp. 417-448 (1973)

18. E.H. Thompson, "A rational algebraic formulation of the problem of the relative orientation", Photogramm. Rec., 3, 14, pp. 152-159 (1959)
19. P.H.S. Torr, D.W. Murray, "The Development and Comparison of Robust Methods for Estimating the Fundamental Matrix", Int J Comput Vision, 24, 3, pp. 271-300 (1997)

20. R.I. Hartley, and A. Zisserman, "Multiple View Geometry", Comp. Vis., Cambridge University (2000)

21. K. Rinner, R. Burkhardt, Photogrammetrie. In Handbuch der Vemessungskunde, (Hsgb. Jordan, Eggert, Kneissel), 3, a/3, Metzlersche Verlagbuchhandlung, p. 2286 (1972)

22. P.J. Rousseeuw, A.M. Leroy, Robust Regression and Outliers Detection, Wiley, New York (1987)

23. G.H. Schut, "On Exact Linear Equation for the Computation of Rotational Elements of Absolute Orientation", Photogrammetria. 17, 1, pp. 34-37 (1961)

24. J. K. Semple, G.T. Kneebone, Algebraic Projective Geometry, Oxford Science (1952)

25. P. Stefanovic, "Relative Orientation - a new approach”, ITC Journal, pp. 417-448 (1973)

26. J. Weng, T.S. Huang, N. Ahuja, Motion and Structures from Image Sequences, Springer (1993)

27. O.D. Faugeras, Q.T. Luong, S.J. Maybank, "Camera self-calibration: Theory and experiments", Proceedings of the $2^{\text {nd }}$ ECCV, pp. 321-334. (1992)

28. M.A. Fischler, R.C. Bolles, "Random Sample Consensus: A Paradigm for Model Fitting with Applications to Image Analysis and Automated Cartography", Comm. of the Ass. for Computing Machinery, 24, pp.381-395 (1981)

29. R.I. Hartley, "Estimation of relative camera position for uncalibrated cameras", Proceedings of the $2^{\text {nd }} E C C V$, pp. 579-587 (1992)

30. R.I. Hartley, "Lines and Points in Three Views - a Unified Approach", Proc. of "Image Understanding Workshop", Monterey, CA, pp. 1009-1016 (1994)

31. I. Niini, "Comparison of the projective block adjustment method versus the bundle method", IAPRS, 33, Part B3, pp. 643-650 (2000)

32. C. Ressl, "An introduction to the relative orientation using the trifocal tensor", IAPRS, 33, Part B3, pp.769-776 (2000)

33. M.E. Spetsakis, J. Aloimonos, "A Unified Theory of Structure from Motion", Proc. of Image Understanding Workshop, Pittsburg, Pennsylvania, pp. 271-283 (1990)

34. P.H.S. Torr, A. Zisserman, S. Maybank, "Robust Detection of Degeneracy", Proceedings of the $5^{\text {th }}$ $E C C V$, Boston, pp. 1037-1044 (1995) 